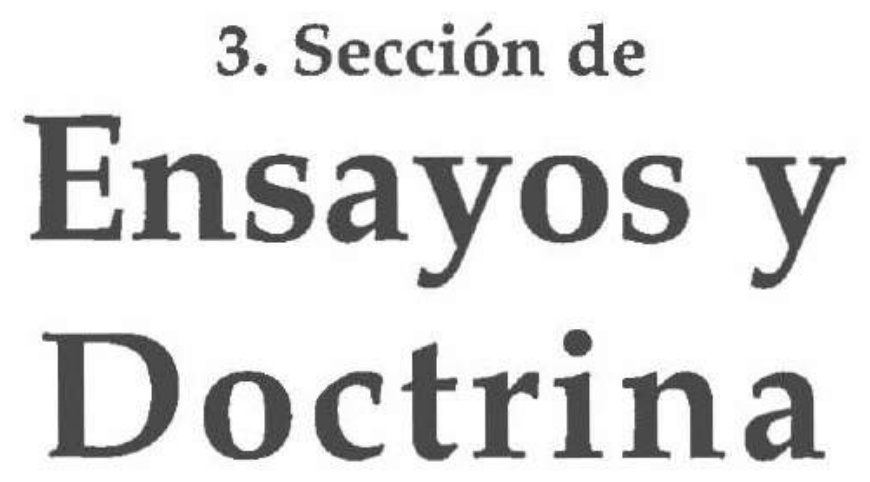





\title{
Reconocimiento y ejecución de LAUDOS EXTRANJEROS anulados: Tendencias globales
}

Anibal Sabater $M$. Efrén C. Olivares ${ }^{1}$

\begin{abstract}
SUMARIO
1. Introducción. 2. La (no) ejecución de laudos extranjeros anulados. 3. Ejecución den caso de laudos extranjeros múltiples y contradictorios. 4. Conclusión.
\end{abstract}

\section{INTRODUCCIÓN}

La Convención de Nueva York de 1958 sobre el Reconocimiento y Ejecución de las Sentencias Arbitrales Extranjeras (la "Convención") -actualmente en vigor en más de 140 países- tiene como objetivo facilitar el reconocimiento y ejecución de laudos extranjeros en los Estados contratantes. Para lograr ese objetivo, la Convención establece, entre otras cosas, siete motivos tasados por los que se puede denegar la ejecución de un laudo extranjero. Sin embargo, la Convención no contiene ninguna norma que establezca los motivos por los cuales se puede anular o denegar la ejecución de un laudo doméstico (o local). Ésta es una cuestión que, en principio, cada Estado con-

1. Anibal Sabater (asabater@fulbright.com) es socio en la oficina de Houston de la firma Fulbright \& Jaworski L.L.P. Efrén C. Olivares (colivares@fulbriglt.com) es abogado asociado en la misma oficina. Ambos se dedican profesionalmente a! arbitraje intemacional. Las opiniones expresadas en este articulo son exclusivamente de los autores y no se corresponden necesariamente con las de otros abogados de su firma. Los autores están a disposición de los lectores que deseen comentar o debatir el contenido de este articulo. 
tratante puede regular libremente. Así, un Estado contratante puede permitir que un laudo doméstico (o sea: un laudo emitido dentro de su territorio) sea anulado por cualquier razón libremente establecida en sus leyes de arbitraje o por la jurisprudencia de sus cortes y tribunales nacionales.

Esta libertad de la que gozan la mayoría de los Estados para gestionar la ejecución y anulación de laudos locales ha generado dos asuntos de interés relacionados con la Convención. Primero, ¿permite la Convención que se ejecute un laudo después de que haya sido anulado en su país de origen? Segundo, ¿cómo deben tratar las cortes de justicia a los laudos que, después de ser ejecutados en el extranjero, resultan anulados o reemplazados en su país de origen? (Siguiendo el texto del artículo V(1)(e) de la Convención, la frase "país de origen" se emplea en estas páginas para referirse al "país en que, o conforme a cuya ley, ha sido dictada esa sentencia".)

El artículo V(1)(e) de la Convención da algunas indicaciones que sientan las bases para responder a estas interrogantes. Según este artículo, "se podrá denegar el reconocimiento y la ejecución de la sentencia [arbitral]" si la parte frente a la que se dirige la solicitud de reconocimiento y ejecución prueba "[q]ue la sentencia [arbitral] no es aún obligatoria para las partes o ha sido anulada o suspendida por una autoridad competente" en su país de origen.

\section{LA (NO) EJECUCIÓN DE LAUDOS EXTRANJEROS ANULADOS}

En la práctica, la frase "se podrá denegar" es crítica para la interpretación del artículo V(1)(e). Para algunos especialistas, el hecho de que el artículo utilice el término potestativo "podrá" -y no el imperativo "habrá de" o "deberá"- significa que los Estados contratantes tienen la facultad, pero no la obligación, de 
denegar el reconocimiento y la ejecución de laudos extranjeros anulados en su país de origen².

En la práctica, algunos juzgados han compartido este punto de vista. Así sucedió, por ejemplo, en 1996 en el llamado "caso Chromalloy" (Matter of Arbitration Betzeen Chromalloy Aeroservices, A Division of Chromalloy Gas Turbine Corp. and The Arab Republic of Egypt, 939 F.Supp. 907 (D.D.C. 1996)). Chromalloy, una corporación estadounidense, celebró en 1998 un contrato con Egipto para la prestación de servicios a los helicópteros de la Fuerza Aérea Egipcia. A finales de 1991 Egipto anunció que daba por terminado el contrato. A continuación, y de conformidad con el contrato, se inició en El Cairo un arbitraje, en el que Chromalloy obtuvo un laudo favorable. Posteriormente, Egipto impugnó el laudo ante la Corte de Apelación de Egipto, mientras Chromalloy intentaba ejecutarlo en una Corte Federal de Distrito de Estados Unidos 3 . La Corte egipcia suspendió y a la postre anuló el laudo porque, según la Corte, los árbitros habían aplicado incorrectamente el derecho civil en lugar del derecho administrativo egipcio. Egipto pidió a continuación la desestimación de la acción de ejecución que aún estaba pendiente en Estados Unidos.

En su fallo, la Corte de Distrito estadounidense afirmó que el artículo $\mathrm{V}(1)(\mathrm{e})$ le confería discrecionalidad para decidir si ejecutaba o no un laudo extranjero anulado. La Corte de Distrito también analizó el artículo VII de la Convención, que establece que

"Las disposiciones de la presente Convención no... privarán a ninguna de las partes interesadas de cualquier derecho

2. Ver PAut.Sson, Jan. "May or Must Under the New York Convention: An Exercise in Symtax and Linguistics". En: Arbitrution International. 1998, Vol. 14.2, p. 229.

3. El sistema judicial federal estadounidense está compuesto, en tèrminos generales, por cortes de distrito, de circuito, y la Suprema Corte. Las contes de distrito son de primera instancia, y cada entidad federativa cuenta con uno o más distritos (c.g., el Distrito del Este de Nucva York, el Distrito del Sur de California, etc.). Las cortes de circuito son cortes de apelación. Existen doce circuitos (once, más la Corte del Circuito de Washington, D.C.), y estain organizadas gcográficamente sin que ningún circuito sea superior a otro. Los fallos de las cortes de circuito pueden apelarse a la instancia final, la Suprema Corte de los Estados Unidos. 
que pudiera tener a hacer valer una sentencia arbitral en la forma y medida admitidas por la legislación ... del país donde dicha sentencia se invoque".

Basándose en el artículo VII, la el juzgado estadounidense concluyó que el reconocer y dar efecto a la anulación del laudo ordenada por la Corte egipcia -y, por tanto, el denegar la ejecución del laudo egipcio- sería una decisión contraria al arbitraje como tal y, por lo tanto, a los principios generalmente aplicados por las cortes estadounidenses. Después de llevar a cabo un extenso análisis de la sentencia dictada por la Corte egipcia, el juzgado estadounidense también concluyó que la defectuosa aplicación del derecho egipcio en el laudo, de haberse realmente producido, no estaba sujeta a revisión por las cortes egipcias, era irrelevante a efectos prácticos y no debería llevar a un cambio en el fallo o resolución contenida en el laudo. Por último, la Corte de Distrito hizo énfasis en que Egipto había incumplido su acuerdo contractual de no apelar la sentencia arbitral. Por estas razones, la Corte falló que la decisión egipcia anulando el laudo no tenía efecto de cosa juzgada. El laudo original de los árbitros a favor de Chromalloy fue, así pues, confirmado y ejecutado en los Estados Unidos.

La decisión judicial estadounidense en el caso de Chromalloy fue recibida con cierta polémica" y ha sido cuestionada hasta el punto de que, en varios casos posteriores los juzgados de Estados Unidos, han rechazado la posibilidad de ejecutar laudos extranjeros anulados. En el caso Baker Marine, por ejemplo, el Segundo Circuito denegó la ejecución de un laudo arbitral que había sido anulado en Nigeria, la sede del arbitraje (Bnker Marine (Nig.) Ltd. v. Chevron (Nig.) Ltd., 191 F.3d 194 (2d Cir. 1999)). En el centro de la controversia en Baker Marine estaba un contrato que estipulaba que Baker Marine y Danos habian de prestar ciertos servicios marítimos a Chevron en Nigeria. De

4. Icr eg., Gharavi, H.G. "The Legal Inconsistencies of Chromalloy". En: Mealev s Internutional Arbitration Report, mayo de 1997, p. 21, PAuLsson, Jan. "Rediscovering the N.Y. Convention: Further Reflections on Chromalloy", En: Afactev's International Arhitration Report, abril de 1997, p. 26. 
acuerdo con la cláusula arbitral, las partes iniciaron el procedimiento de arbitraje en Lagos, Nigeria, bajo las reglas de arbitraje de la CNUDMI/UNCITRAL y la ley de Nigeria.

Baker Marine obtuvo dos laudos favorables en contra de Chevron y Danos, y posteriormente intentó ejecutar ambos ante la Corte Superior Federal de Nigeria, que anuló ambos por considerarlos contrarios al derecho nigeriano. Entre otras cosas, la Corte nigeriana afirmó que el laudo contra Danos no tenía fundamentos probatorios y que la indemnización punitiva contenida en el laudo contra Chevron era inapropiada. A continuación, y a pesar de su anulación en el Estado de origen, Baker Marine intentó ejecutar los laudos en una Corte Federal en Nueva York. La Corte federal, sin embargo, denegó la ejecución de los laudos basándose en principios de igualdad o paridad internacional, los cuales, según la Corte, requieren que se respete la decisión de la autoridad judicial competente en la sede del arbitraje.

Baker Marine apeló el fallo. En segunda instancia, el Segundo Circuito explicó las razones por las que consideraba que Baker Marine era un caso distinto a -y que no podía ser resuelto igual que- Chromalloy. Entre otras razones, para el Segundo Circuito, en Baker Marine (a diferencia de en Chromalloy) las partes no habían renunciado a impugnar el laudo. Además, la Corte del Segundo Circuito rechazó que tuviera la potestad de revisar el derecho nacional nigeriano y de verificar el modo en el que éste había sido aplicado por el juzgado nigeriano que anuló el laudo (en Chromalloy, la corte estadounidense había afirmado exactamente lo contrario). Además, la Corte del Segundo Circuito afirmó que, dado que Baker Marine no había expuesto motivos de entidad para no acatar el fallo de la Corte nigeriana, la anulación del laudo en Nigeria debía de ser respetada. En vista de todo lo anterior, el Segundo Circuito confirmó el fallo de la Corte de Distrito que denegaba la ejecución del laudo en Estados Unidos. 
Al día de hoy, la solución adoptada en Baker Marine parece haber ganado terreno en las cortes estadounidenses, mientras que Chromalloy, al parecer, sigue siendo la excepción.

Recientemente, una Corte del Circuito de Washington, D.C. aplicó el razonamiento del caso Baker Marine para resolver el llamado "caso TermoRio" (TermoRio S.A. E.S.P. v. Electranta S.P., 487 F.3d 928 (D.C. 2007), cert. denied, 128 S.Ct. 650 (2007)). Electranta, una empresa paraestatal colombiana, acordó comprar energía eléctrica a TermoRío de conformidad con lo dispuesto en un contrato de suministro de energía eléctrica. Después de que Electranta supuestamente incumpliera con sus obligaciones, las partes iniciaron un arbitraje en Colombia bajo las reglas de la Cámara de Comercio Internacional ("CCI"), según se estipulaba en el contrato. El Tribunal emitió su laudo a favor de TermoRío. Posteriormente, Electranta lo impugnó en los tribunales colombianos. La Sala de lo Contencioso Administrativo del Tribunal Supremo de Colombia anuló el laudo sosteniendo que éste violentaba el derecho colombiano, ya que no permitía explícitamente el arbitraje bajo las reglas de la $\mathrm{CCI}-\mathrm{o}$, en realidad, bajo ningún otro tipo de arbitraje internacional institucional- a la fecha del contrato.

$\mathrm{Al}$ igual que en Baker Marine, TermoRío intentó ejecutar el laudo en una Corte Federal de Estados Unidos. La Corte desestimó la acción. En el subsecuente recurso de apelación, la Corte de Circuito de Washington, D.C. resolvió que, de un lado, el laudo había sido "anulado conforme a derecho, por el pais donde éste fue emitido" 5 , de otro y en consecuencia, procedía desestimar, al amparo del artículo V(1)(e) de la Convención, la acción de ejecución presentada por TermoRío: "IN]o existe laudo arbitral ... si éste ha sido anulado conforme a derecho por la autoridad competente en el Estado donde éste fue emitido. Este principio controla la resolución de este caso" "6. Aun así, la Corte estadounidense que resolvió el caso

5. "[L]awfully nullified, by the country in which the award was made". Todas las traducciones son de los autores.

6. "An arbitration award does not exist ... if it has been lawfully 'set aside' by a competent authority in the State in which the award was made. This principle controls the disposition of this case". 
TermoRio reconoció el precedente sentado por Chromalloy y aceptó que existe un "fino manto de orden público sobre el Artículo $V(1)(e), "$ " que permitiría ordenar la ejecución de un laudo extranjero si éste ha sido anulado en su país de origen por razones que ofenden las nociones fundamentales de decencia y de justicia. En otras palabras: aunque el fallo en los tres asuntos pudiera diferir, las cortes estadounidenses parecen haber aceptado en Chromalloy, Baker Marine y TermoRío que la anulación de un laudo en su país de origen no acarrea necesaria e ineludiblemente la anulación del laudo y está sujeta a revisión y escrutinio en el país extranjero en el que se trata de ejecutar el laudo.

Poco tiempo después del fallo en TermoRío, una corte en Rubí, España, intentó definir las circunstancias en las que se puede ejecutar un laudo, cuando éste haya sido anulado en su país de origen. En concreto, la Corte de Primera Instancia No. 3 de Rubi tuvo que pronunciarse sobre la petición de la parte perdedora en un arbitraje CCI para que se denegara la ejecución de un laudo en España. El laudo había sido dictado en París y se encontraba impugnado en Francia. Según la parte perdedora en el arbitraje, esta circunstancia debía llevar a que el Juzgado de Rubí o bien denegara -de acuerdo con el artículo V(1)(e) de la Convención- la petición de ejecutar el laudo en España, o bien suspendiera -bajo el artículo VI de la Convención- la ejecución del laudo mientras se resolvía el recurso de anulación pendiente ante las cortes francesas. La Corte de Rubí, sin embargo, rechazó ambas peticiones.

Según la Corte de Rubí, la anulación de un laudo en la sede del arbitraje podría impedir su reconocimiento bajo el artículo $\mathrm{V}(1)(e)$ sólo si la anulación está basada en cuestiones jurisdiccionales o en una violación grave al debido proceso. Al determinar que ninguno de estos puntos estaba en controversia en el procedimiento en Francia, la Corte de Rubí ordenó el reconocimiento y ejecución del laudo impugnado en Francia8.

7. "[N]arrow public policy gloss on Article $V(1)(c) "$. 
Además, la Corte de Rubí recordó la importancia del artículo 9 del Convenio Europeo de 1961 sobre Arbitraje Comercial Internacional (el "Convenio Europeo"). Este artículo -como recordó el Juzgado de Rubí- suplementa, en el ámbito europeo, al artículo $\mathrm{V}(1)(\mathrm{e})$ de la Convención. De acuerdo con el artículo 9 del Convenio Europeo, la anulación de un laudo arbitral en el país sede del arbitraje "constituirá causa de denegación en lo referente al reconocimiento o ejecución ... sólo" por las razones enumeradas en los incisos (1)(a)-(d) de este artículo (se le añadió el énfasis). Esas razones enumeradas en los incisos (1)(a)-(d) son muy semejantes a los motivos de denegación de la homologación del laudo extranjero establecidas en el artículo $\mathrm{V}$ de la Convención". Así pues, en Europa, un laudo anulado por motivos distintos de los previstos en el artículo 9 del Convenio Europeo, puede ser ejecutado en el extranjero. En contraste con el Convenio Europeo, la Convención Interamericana sobre Arbitraje Comercial Internacional de 1975 (la "Convención de Panamá") no contiene una disposición que permita alcanzar este resultado y, en su lugar, adopta el texto aparentemente discrecional del artículo V(1)(e) de la Convención de Nueva York, según el cual la corte de turno "podrá" denegar la ejecución si el laudo ha sido anulado en la ciudad o el país sede (Convención de Panamá, artículo 5(1)).

8. Ver "The Enforcement of Annulled Foreign Awards: Worldwide Trends". En: Fulbright af Janvorski International Arbitration Report 2008(2), paigina 9, SABATE R Martí, Anibal. La cficacia en España de los laudes arbitrales cxtranjenos. Madrid: Editorial Tecnos, 2002 (citado en el fallo de Rubi) "[N]arrow public policy gloss on Article V(I)(e)".

9. "1. Sólo se podrá denegar el reconocimiento y la ejecución de la sentencia, a instancia de la parte contra la cual es invocada, si esta parte prucba ante la autoridad competente del pais en que se pide el reconocimiento y la ejecución: (a) Que las partes en el acuerdo a que se refiere el articulo Il estaban sujetas a alguna incapacidad en sirtud de la ley que le es aplicable o que dicho acuerdo no es válido en virtud de la ley a que las partes to han sometido, o si nada se hubiera indicado a este respecto, en virtud de la ley del pais en que se haya dictado la seniencia: o (b) Que la parte contra la cual se invoca la sentencia arbitral no ha sido debidamente notificada de la designación del árbitro o del procedimiento de arbitraje o no ha podido, por cualquier otra razón, hace valer sus medios de defensa; o (c) Que la sentencia se refiere a una difurencia no prevista en el compromiso o no comprendida en las disposiciones de la cláusula compromisoria, o contiene decisiones que exceden de los términos del compromiso o de la cláusula compromisoria, no obstante, si las disposiciones de la sentencia que se refieren a las cuestiones sometidas al arbitraje pueden separarse de las que no lan sido sometidas al arbitraje, se podra dar reconocimiento y cjecución a las primeras; o (d) Que la constitución del tribunal arbitral o el procedimiento arbitral no se han ajustado al acuerdo celcbrado entre las partes 0 , en defecto de tal acuerdo, que la constitucion del tribunal arbitral o del procedimiento arbitral no se han ajustado a la ley del pais donde se ha efectuado el arbutraje...". Convención, articulo V(1)(a)-(d). 


\section{EJECUCIÓN EN CASO dE laUdos EXTRANJEROS MÚLTIPLES Y CONTRADICTORIOS}

Para complicar aún más la jurisprudencia en torno al artículo V(1)(e) de la Convención, existe la posibilidad de laudos contradictorios. Ni la Corte en TermoRío ni la de Rubí contemplaron esta posibilidad, que consiste en que el laudo sea anulado o reemplazado en su país de origen, pero después de que haya sido completamente ejecutado en el extranjero. Esto fue precisamente lo que sucedió en el llamado "caso Hilmarton", en el que un laudo suizo fue anulado y reemplazado por otro incompatible con él después de que el primer laudo hubiera sido plenamente ejecutado en Francia. Cuando se produce una situación así, las cortes se enfrentan el dilema de reconocer el segundo laudo y anular todo lo relativo a la ejecución del primero, o respetar el reconocimiento y ejecución del primero, bajo el principio de la cosa juzgada.

Albert Jan VAN DEN BerG anticipó este problema en 1981 en sus comentarios a la Convención: "Si ocurriese una anulación subsecuente, pareciera razonable cancelar la orden que concedió la ejecución [del laudo]. La cuestión tendría que resolverse en base a lo dispuesto por el artículo III de la Convención bajo la ley procesal del país donde se otorgó la ejecución"10. De hecho, así es como la ley de arbitraje de Alemania de 1998 ha resuelto el problema: autorizando a las cortes de justicia a denegar la ejecución de un laudo extranjero previamente reconocido si éste ha sido anulado en su país de origen después de su reconocimiento en el extranjero (\$ 1061(3) ZPO Ausländische Schiedsspriïhe).

Sin embargo, en Francia, la Corte Suprema ha tomado una postura distinta en los casos Hilmarton (1994) y Renn Holding (2007). En ambos casos, la Corte francesa rehusó "cancelar" la

10. "If a subsequent setting aside occurs, it would seem to be reasonable to cancel the order by which enforcenent is granted. The question would seem to have to be solved by virtue of Article III of the Convention under the law of the procedure of the country where enforcement is granted". 
ejecución del primer laudo, a pesar de que éste hubiera sido anulado en su país de origen.

En Hilmarton, OTV, la parte vencedora en un primer arbitraje en Suiza, logró ejecutar el laudo en Francia luego de que éste había sido revocado por una corte suiza y de que la corte suiza hubiera ordenado al tribunal que dictara un nuevo laudo"1. Cuando el tribunal arbitral suizo emitió un segundo laudo en el mismo caso, esta vez a favor de Hilmarton, Hilmarton trató de ejecutarlo en Francia. La Corte de Apelación de Versalles reconoció el segundo laudo'2, pero, a continuación, la Corte Suprema de Francia revocó la decisión de Versalles y falló que el primer laudo tenía efecto de cosa juzgada (res iudicata), lo cual hacía improcedente el reconocimiento y ejecución del segundo ${ }^{13}$.

Recientemente, la Suprema Corte de Francia adoptó una solución similar en el caso de Rena Holding (PT Putrabali Adyamulia v. Rena Holding, 29 de junio de 2007, casos nos. 0613.293 y 05-18.053). La empresa indonesa PT Putrabali le había vendido especias a la sociedad francesa Société Est Epices (que luego se convertiría en Rena Holding). Las especias se perdieron en altamar a causa de un choque entre buques. Rena rehusó pagar por las especias, y Putrabali inició procedimiento de arbitraje en Londres ante la Asociación General Internacional de Productos Alimenticios (International General Produce Association).

Rena se impuso en este primer arbitraje. Sin embargo, un juez en Inglaterra revocó parcialmente el laudo y lo reenvió al tribunal arbitral. Posteriormente, el tribunal emitió un segundo laudo, esta vez a favor de Putrabali, ordenando que Rena Holding efectuara el pago. Para entonces, sin embargo, Rena Holding ya había conseguido ejecutar el primer laudo en

11. Société Hilmarton Lid. v. Société OTV (Cour de cassation, 23 de marzo de 1994), 1994 Revue de L'Arbitrage 327, traducido en XX Y.B. Com. Arb. 663 (1995).

12. Société OTV s: Socièté Hilmarton (Cour d'appel de Versailles, 29 de junio de 1995), traducido en XXI Y.B. Com. Arb. 524 (1996).

13. Société OTV v Société Hilmarton (Cour de cassation, 1re Ch. Civ., 10 de junio de 1997), XXII Y.B. Com. Arb. 696 (1997). 
Francia ${ }^{14}$. A la postre, la Corte Suprema de Francia consideró el segundo laudo no-ejecutable, ya que la ejecución del primero tenía efectos de cosa juzgada y preclusivos. La Corte Suprema afirmó que la ejecución del primer laudo en Francia era correcta y no debía ser modificada porque, según su parecer, los laudos arbitrales son autónomos y su destino no está anudado a las vicisitudes que atraviesen en el país de origen. La Corte Suprema francesa también afirmó que el artículo VII de la Convención (transcrito más arriba) habilita a las cortes francesas a aplicar el derecho interno francés cuando ese resultado es más favorable a la ejecución del laudo. Según la Corte Suprema francesa, el derecho interno francés no incluye la anulación en el país de origen entre los motivos para denegar su ejecución (artículo 1502 del Nouvenu Code de procedure civile) ${ }^{15}$.

El fallo en Rena Holding se enfrenta a un problema que las cortes intentaron evadir en Baker Marine y TermoRío. Parte del razonamiento en Baker Marine (del que luego se hizo eco TermoRio) fue que "la aplicación mecánica del derecho arbitral nacional [del lugar de la ejecución] a los laudos extranjeros... atentaría seriamente contra la finalidad de éstos y produciría laudos contradictorios [con lo resuelto en el pais de origen]"16.

Sin embargo, la aplicación del derecho arbitral nacional (que es lo que permitieron los jueces de ejecución en los casos Hilmarton y Renn Holding) no es la única manera de producir fallos contradictorios. Aún si las cortes del lugar de ejecución difieren la decisión sobre la validez y efectos del laudo a los jueces del país de origen, esto no resuelve el problema de la finalidad de los laudos, ya que éstos pueden ser anulados en el país sede del arbitraje con posterioridad a su ejecución en otro país.

14. Societé PT Putrabali Adyamulia v: SA Rena Holding et autre (Cour d'uppel de Paris, Ire Ch. Civ, 31 de marzo de 2005), 2005 Revue de L'Arbitrage, No. 3, p. 792.

15. Socicté PT Putrabali Adyamulia b. Société Rena Holding et Socićte Mogunta Est Epices (Cour de cassation, 29 de junio de 2007, casos nos. 06-13.293 y 05-18.053 \}, 2007 Revue de L'Arbitrage No. 3. p. 645.

16. "[M]echanical application of domestic arbitral law to forcign awards under the Convention would seriously undermine finality and regularly produce conflicting judgments". 


\section{ConClusión}

Las cortes pueden insistir en que la decisión sobre si un laudo existe y es ejecutable corresponden en exclusiva a los jueces del país de origen y debe ser necesariamente acatada por todos los jueces extranjeros de ejecución. En su defecto, se puede reconocer que es posible que existan pronunciamientos contradictorios en distintos países sobre la validez y ejecución de un laudo. Ninguna solución está exenta de dificultades prácticas. En todo caso, las partes en un contrato deben considerar muy cuidadosamente la sede del arbitraje en su cláusula arbitral, ya que ésta puede llegar a tener consecuencias importantes a la hora de ejecutar un laudo. 